\title{
Application-Aware Protection in DWDM Optical Networks
}

\author{
Hamza Drid, Bernard Cousin \\ University of Rennes I-IRISA (INRIA of Rennes) \\ Campus de Beaulieu, \\ 35042 Rennes Cedex, France \\ Email: \{hdrid, bcousin\}@irisa.fr
}

\author{
Nasir Ghani \\ ECE Department, University of New Mexico \\ Albuquerque, NM 87111, USA \\ Email: nghani@ece.unm.edu
}

\begin{abstract}
Fast recovery time and reduced resource utilization are the two main criteria for determining the quality of survivability mechanism. Now it is well-known that link-based protection and path-based protection provide respectively a short recovery time and reduced use of resources. To benefit from the both of these saliencies, we propose in this paper to use these mechanisms simultaneously. Indeed, demands mandating shorter recovery time will be protected using link-based protection. Meanwhile other demands will be protected using pathbased protection. Simulation results show that the proposed solution achieves a good trade-off between resource utilization and recovery time.
\end{abstract}

\section{INTRODUCTION}

Survivability means that the network has the ability to maintain acceptable levels of service even after an occurrence of failures within the network. Now given the everincreasing speed of modern optical dense wavelength division multiplexing (DWDM) backbones, failure events lasting few seconds may cause massive losses, i.e., both in terms of data volumes and ensuing revenue declines. Therefore it is crucial to develop rapid survivability mechanisms that work to minimize the level of damage. Furthermore, given that the number of wavelengths channels may be limited in most DWDM networks (and new fiber build-outs are timely and costly), related survivability mechanisms should also minimize their overall resources usages. As a result, these two criteria-fast recovery and resource minimization-form the key objectives for ascertaining the quality of survivability mechanisms in this effort.

Now most optical network survivability schemes can generally be classified into one of two key categories, protection[1] or restoration[2]. Namely, restoration is a reactive approach in which a backup light-path connection is searched and established after a failure on the primary light-path occurs. Meanwhile, protection is a pro-active approach in which the backup light-path is pre-reserved, i.e., at the same time with the working light-path setup. Hence these mechanisms can guarantees full recovery whereas restoration schemes cannot. Overall, since failures may result in large losses, protection schemes are generally favored in optical DWDM networks.

To date, various protection mechanisms have been studied for DWDM networks, including link-based [3] and path-based
[4]. Namely, link-based protection provides a backup for each link of a primary light-path. Hence, upon failure of a link, the end-nodes of the failed link activate the backup path and reroute the traffic around the failed link. Meanwhile, in pathbased protection, only one backup light-path is computed to protect all links in the primary light-path. Hence when a link fails here, notification messages are sent to the source and destination nodes in order to activate and reroute the traffic on the backup light-path, i.e., switchovers.

From the above, it is noted that path-based protection is usually more efficient in terms of capacity utilization, as compared to the link-based protection, i.e., since only one backup light-path is required to protect all links in the primary light-path. Indeed, many studies have already shown this result [8]. In addition, path-based approach can also protect all nodes in the primary light-path, except the source and destination nodes. By contrast, link-based protection is generally more resource-intensive and only provides "localized" protection. However, at the same time, this approach gives much faster recovery times, i.e., since only the two end-point nodes of the failed link are involved in the recovery switchover.

In order to leverage the saliencies of both link- and pathbased protection mechanisms, in this effort we propose to use them simultaneously. Specifically, the exact choice of protection mechanism will be made according to the user application demand requirements. Namely, demands requiring very fast recovery times will be provisioned with linkbased protection, whereas other demands will be protected using path-based strategies. Overall, this "joint" solution tries to achieve an acceptable tradeoff between two competing objectives, i.e., minimizing backup resource efficiencies and minimizing recovery timescales.

Overall, this paper is organized as follows: First, Sections 2 and 3 present our motivations and the description of our heuristic scheme, respectively. Next, Section 4 studies the performance of the scheme using network simulation. Finally, conclusions and directions for future work are presented in Section 5.

\section{Motivation}

In this section we describe the key motivation of our approach. Specifically, the requirement needs of some of today's 
applications are analyzed, and then the main guidelines of our heuristic are introduced to meet those requirements.

Over the past years, the variety and number of highbandwidth network applications has grown significantly. For some applications, a failure in network connectivity can disrupt a mission-critical transaction, which in turn can even be catastrophic, e.g., remote instrument operation, telemedicine/surgery, etc. In general, these applications are classified here as "real-time" applications, and are characterized by hard real-time requirements, e.g., guaranteed bandwidth, very low delay, minimal loss. Meanwhile, there are also various other applications that can tolerate a certain amount of time delay, as long as loss behaviors do not occur, e.g., such as e-mail, chat applications, remote backup/storage, etc. Furthermore, application demands can also be classified based upon their nature, e.g., critical and no-critical. Here, the former types require very short recovery times, whereas the latter types can generally suffice with somewhat more latent recovery.

Using these above classifications, we now propose a novel heuristics-based solution for protecting critical demands using link protection. As a result, the associated recovery times here will be significantly reduced here. Meanwhile, demands with less stringent requirements will be protected using path protection, thereby providing an improved level of resource efficiency in the network.

\section{Heuristic SCHEME}

The proposed "application-aware" protection scheme is now presented here.

\section{A. Network model}

Consider the requisite notation first. The DWDM optical network is modeled as an undirected graph $G=(V, E)$, in which each optical cross-connect (OXC) node $v \in V$ represents an optical switch and each edge $e \in E$ represents a network link. Here, all links are assumed to be bi-directional and also contain $W$ available wavelengths. Furthermore, is assumed that each user demand requires one optical lightpath connection across the network, albeit this can readily be generalized to handle multiple wavelengths as well. Finally, it is also assumed that each $O X C$ node has full wavelength conversion capability, thereby precluding the further consideration of wavelength selection in this current effort. Now before presenting the details of the heuristic scheme, some additional variables are also introduced as follows:

$C_{i}$ : Cost of link $\mathrm{j}$; it depends of physical length, installation cost, etc.

$C r_{j}$ : Current cost of link j given as follows (Eq. 1):

$$
C r_{j}= \begin{cases}\frac{W-F_{j}}{W}+C_{i} & \text { if } F_{j}>0 \\ \infty & \text { otherwise }\end{cases}
$$

$F_{j}$ : Number of available wavelengths on link $j$

$B_{j}$ : Number of backup wavelengths on link $j$
$D_{n}:$ Demand number $n$

Using the above, the proposed scheme implements the following set of steps:

- Step 1: Loop and wait for demand arrival. If a demand $D_{n}$ arrives, go to Step 2 .

- Step 2: Adjust the link-cost according to Eq. 1 and compute the shortest-route from the source node $s$ to destination node $d$ as the working path $W P_{n}$. If $W P_{n}$ can be found successfully, go to Step 3; otherwise, block demand, restore the network state, and go back to Step 1.

- Step 3: if $D_{n}$ is a critical request go to Step 5; otherwise go to Step 4.

- Step 4: Compute the shortest-path from the source node $s$ to destination node $d$ as the backup path $B P_{n} . W P_{n}$, and $B P_{n}$ should be disjoint. If $B P_{n}$ can be found successfully, accept this demand, update the network state in $G=(V, E)$, and go back to Step 1; otherwise, block this demand, restore the network state, and go back to Step 1. (i.e., Step4 $\Leftrightarrow$ path-based protection)

- Step 5: For each link of working path $W P_{n}$, compute a shortest-path as the backup path $B P_{n, i}$. If all backup paths $B P_{n, i}$ are found successfully, accept this demand, update the network state in $G=(V, E)$, and go back to Step 1; otherwise, block this demand, restore the network state, and go back to Step 1. (i.e., Step5 $\Leftrightarrow$ link-based protection)

\section{PERFormanCE VAlUATION}

The performance of the proposed "application-aware" heuristic algorithm is now analyzed using discrete event simulation. Specifically, the key evaluation metrics used here include resource utilization and recovery time. In particular, simulation experiments are carried out using two widely-used network topologies (US network [9] and NSF network [10]) shown in Fig. 1. In each of these networks, it is assumed that each fiber link has $W=32$ wavelengths and all OXC nodes support full wavelength conversion.

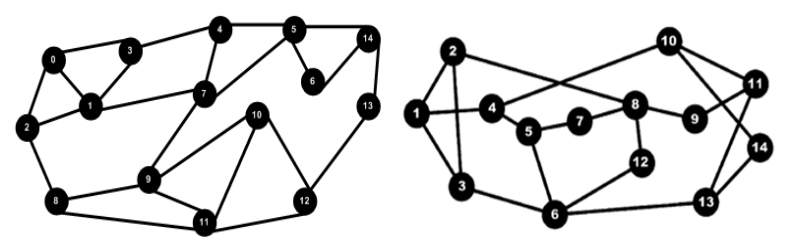

Fig. 1. Test networks 
Meanwhile, the demand traffic model used in our simulations is the incremental traffic model of [5], in which connection requests (for a random source and destination) enter the network sequentially. Once a connection request is satisfied, it is assumed that its lightpath remains indefinitely in the network, i.e., it is never released. Indeed, this is quite representative of traffic on most real-world optical backbones, and moreover, is somewhat simpler than other dynamic traffic profiles used for testing "on-line" provisioning heuristic algorithms. As stated earlier, it is also assumed that each demand requests one wavelength unit of capacity. Finally, there are no waiting queues for network requests, i.e., subsequent retries of failed demands are not allowed and such requests are simply rejected.

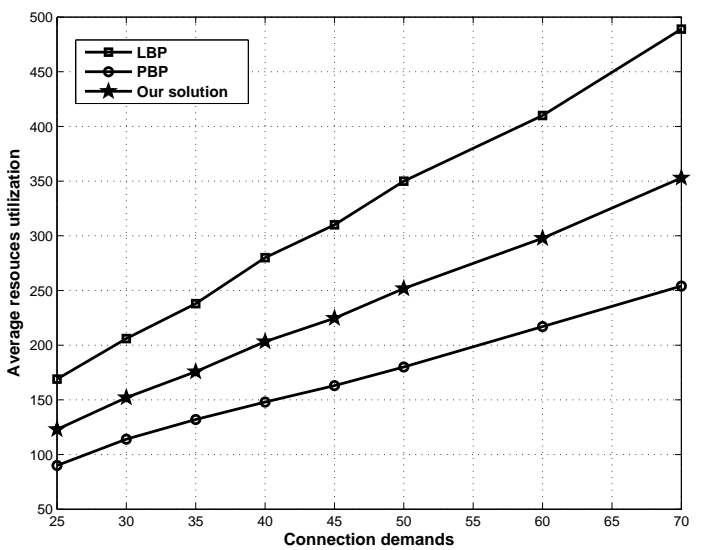

Fig. 2. Resource utilization (NSF network)

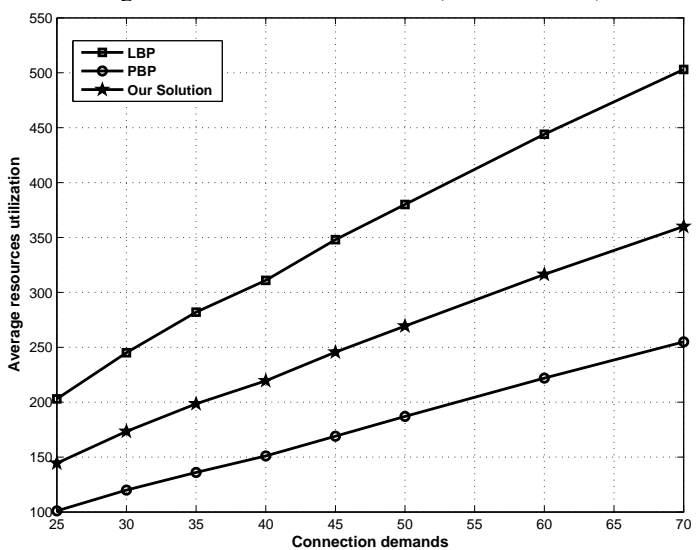

Fig. 3. Resource utilization (US network)

To evaluate our solution, three different scenarios are considered. Namely, in the first scenario path-based protection (PBP) is implemented, in which one backup light-path is computed to protect all links in the primary light-path. Meanwhile, in the second scenario, link-based protection (LBP) is used, where a backup lightpath is computed for each link in a primary light-path. At last in the third scenario, the simultaneous path- and link-based protection schemes are implemented, as per differing demand requirements. Here we distinguish between two kinds of demands, critical and no- critical. Specifically, these demands are randomly generated using a uniform distribution, i.e., with 50\% selection of each demand type.

Finally, the key performance metrics used include resource utilization (RU)[6] and recovery time(RT)[7]. The former gives insights into the quality of protection and is defined as the sum of the total backup and primary wavelength resources used. Hence low RU is more efficient than high RU due to the fact that high RU requires a large capacity to establish and protect the connection against failures. The average RU values as a

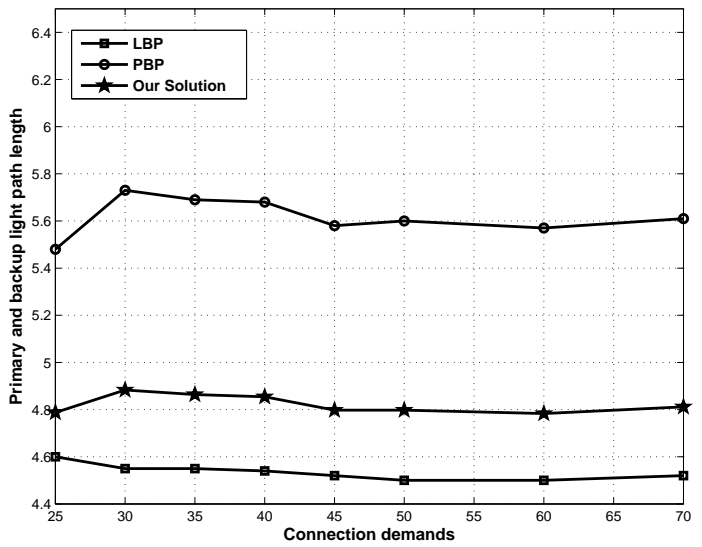

Fig. 4. Recovery time (NSF network)

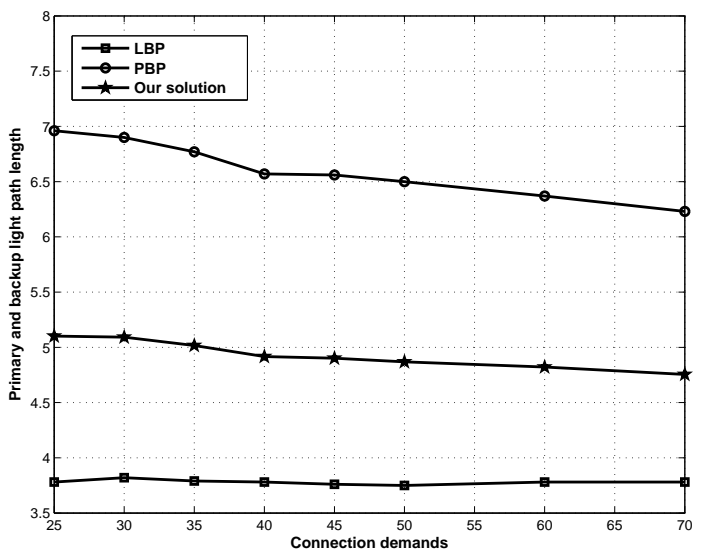

Fig. 5. Recovery time (US network)

function of number of requests (load) is plotted in figure 2 and 3. Here it is clear that PBP protection is better than the LBP protection. The reason for this is that link-based protection assigns for each link of primary light-path a backup light path while path-based protection use only one backup light-path to protect all links in the primary light-path. Meanwhile the proposed joint scheme achieves a tradeoff between the two, as expected.

The corresponding recovery times for these schemes are also analyzed here. Now this value typically depends upon the length of the primary and backup light-paths [7,9], i.e., shorter light-path lengths lead to a faster recovery times. Hence in our simulations, the recovery time is gauged as the average length of primary and backup light-paths. The overall results here 
are again shown for varying connection demands in figure 4 and 5. As expected, link-based protection is faster because only the two end nodes of the failed link need to perform restoration. Furthermore, simulations show that the proposed joint solution achieves an acceptable tradeoff between two competing objectives, i.e., minimizing backup resource and minimizing recovery timescales. This tradeoff is due to the exploitation of link and path protection advantages.

\section{CONCLuSion}

This paper studies survivability in WDM optical networks and focuses on two key objectives of resource minimization and recovery times. The relative merits of path- and linkbased protection are discussed and then a joint scheme is developed to incorporate both. Namely, we propose to protect critical demands using link-based protection and thereby reduce recovery times. At the same time, we propose to protect less stringent demands with path-based protection, thereby reducing resource utilizations.

Overall, our results show that the joint solution achieves a good tradeoff between two competing goals: efficient use of backup resources and short recovery time. This study is very encouraging, and we intend to continue our research by using other kind of networks such as multi-domain optical networks.

\section{REFERENCES}

[1] S. Ramamurthy and B. Mukherjee, Survivable WDM mesh networks- Part I: Protection, Proc. IEEE INFOCOM, Mar. 1999, pp. 744-751.

[2] S. Ramamurthy and B. Mukherjee, Survivable WDM mesh networksPart II: Restoration, Proc. IEEE Integrated Circuits Conf., June 1999, pp. 2023-2030.

[3] P. Ho, J. Tapolcai and T. Cinkler, Segment shared protection in mesh communications networks with bandwidth guaranteed tunnels, IEEE/ACM Transactions on Networking, 2004, pp.1105-1118.

[4] H. Zang, C. Ou and B. Mukherjee, Path-protection routing and wavelength assignment (RWA) in WDM mesh networks under duct-layer constraints, IEEE/ACM Transactions on Networking, 2003, pp. 248-258.

[5] D. Eric et al., Deogun On The Bandwidth Efficiency of PreCrossconnected Trails. Proc. of IEEE International Conference on Communications, 2007, pp. 2294-2299.

[6] H. Drid, B. Cousin, S. Lahoud and M. Molnór, Multi-criteria p-cycle network design, IEEE Conference on Local Computer Networks, Montreal, Canada, Oct 2008, pp. 361-366.

[7] H. Drid, B. Cousin, S. Lahoud and M. Molnór, A survey of survivability in multi-domain optical networks, Computer Communications, 2010, pp. 10051012.

[8] M. Guido, et al., Optical Network Survivability: Protection Techniques in the WDM Layer, Photonic Network Communications, 2002, pp. 251-269

[9] X. Zhang, D. Liao, S. Wang and H. Yu, On segment shared protection for dynamic connections in multi-domain optical mesh networks, International Journal of Electronics and Communications, 2009, pp. 1-6.

[10] F. Zhang, and W. Zhong,Performance Evaluation of Optical Multicast Protection Approaches for Combined Node and Link Failure Recovery, Journal Of Lightwave Technology, 2009, pp. 4017-4025. 